\title{
Effects of Saikosaponin D on apoptosis in human U87 glioblastoma cells
}

\author{
YAN LI ${ }^{1}$, TONGJIAN CAI ${ }^{2}$, WEI ZHANG ${ }^{1}$, WENHE ZHU ${ }^{1}$ and SHIJIE LV $^{1}$ \\ ${ }^{1}$ Department of Biochemistry, Jilin Medical University, Jilin, Jilin 132013; ${ }^{2}$ Department of Epidemiology, \\ College of Preventive Medicine, Third Military Medical University, Chongqing 400038, P.R. China
}

Received June 27, 2016; Accepted May 31, 2017

DOI: $10.3892 / \mathrm{mmr} .2017 .6765$

\begin{abstract}
Saikosaponin D (SSd) is a type of Saponin derivative, which is a component extracted from Bupleurum falactum. SSd has been reported to exert anticancer activities. However, the effects of SSd on gliomas have not been elucidated. The aim of the present study was to investigate the pharmacological functions and potential molecular mechanisms of SSd in human U87 glioblastoma cells. The cells were treated with SSd at various concentrations for $48 \mathrm{~h}$, the cell viability was assessed with 3-(4,5-dimethylthiazol-2-yl)-2,5-diphenyltetrazolium assay, and the activation of Akt, extracellular signal-regulated kinases (ERK), c-Jun N-terminal kinases (JNK) and caspase-3 was assessed by western blotting. In addition, apoptosis levels were analyzed with Hoechst 33258 and Annexin V staining. The results demonstrated that treatment of the U87 glioma cells with SSd markedly suppressed cell proliferation in a dose-dependent manner. Meanwhile, SSd treatment enhanced apoptosis in the U87 cells. Furthermore, SSd significantly inhibited the phosphorylation of Akt and ERK, and promoted phosphorylated-JNK and cleaved caspase-3 expression. The present study revealed the potential therapeutic effects of $\mathrm{SSd}$ in the treatment of gliomas, and the cytotoxic effects of SSd in U87 cells were at least partly attributed to the depression of phosphatidylinositol 3-kinase/Akt and ERK protein expression levels, and activation of JNK and caspase-3 expression.
\end{abstract}

\section{Introduction}

Gliomas are the most common and most fatal tumors of all types of brain malignancy $(1,2)$. The general treatment measures against gliomas include surgery, radiotherapy and chemotherapy; however, the prognosis is poor, and the median

Correspondence to: Professor Shijie Lv, Department of Biochemistry, Jilin Medical University, 5 Jilin Road, Jilin, Jilin 132013, P.R. China

E-mail: jlmpclvsj@163.com

Key words: saikosaponin D, glioma, Akt, extracellular signal-regulated kinases, c-Jun N-terminal kinases, caspase-3, apoptosis survival time of gliomas is only 6-14 months (3-5). Gliomas are often resistant to antitumoral chemotherapeutic strategies, thus limiting the efficacy of treatment (4). The pharmacological activities of traditional Chinese medicines have attracted increasing attention regarding their therapeutic effects against glioblastoma (6-8).

Saikosaponin D (SSd) is one of the major Saponin components derived from the dried roots of Bupleurum falactum, a traditional Chinese medicine plant. It has been reported that SSd exerts anticancer activities in cervical and lung cancer cells, and hepatocellular carcinoma cells (9-12). SSd may potentiate tumor necrosis factor (TNF)- $\alpha$-mediated cell death via suppression of TNF- $\alpha$-induced nuclear factor (NF)- $\kappa \mathrm{B}$ activation, while inducing apoptosis by enhancing the loss of mitochondrial membrane potential (13). In addition, SSd has been demonstrated as an inhibitor of cell survival signaling, and subsequently attenuates the expression of B-cell lymphoma-extra large (14). However, the molecular mechanisms remain unknown.

Although previous studies have indicated that SSd exerts anticancer activities in various tumor cell lines (9-12), the effects of it in central nervous system (CNS) malignant tumors remain unknown. In the present study, the growth potential, proliferation inhibition and apoptosis induction effects of SSd on human U87 glioma cells were investigated. In addition, the possible mechanisms underlying SSd-induced growth arrest and apoptosis in glioma cells were evaluated.

\section{Materials and methods}

Chemicals and reagents. SSd (purity, 96\%) was purchased from the National Institutes for Food and Drug Control of China (Beijing, China). The 3-(4,5-dimethylthiazol-2-yl)-2,5-diphenyltetrazolium (MTT) was obtained from Sigma-Aldrich (Merck KGaA, Darmstadt, Germany). Dulbecco's modified Eagle's medium (DMEM) was purchased from Gibco (Thermo Fisher Scientific, Inc., Waltham, MA, USA). Akt (cat. no. 4691), phosphorylated (p)-Akt (cat. no. 4060), extracellular signal-regulated kinases (ERK; cat. no. 4695), p-ERK (cat. no. 4370), c-Jun N-terminal kinases (JNK; cat. no. 9258), p-JNK (cat. no. 4668), cleaved caspase-3 (cat. no. 9664), $\beta$-actin (cat. no. 12620) and peroxidase-conjugated anti-rabbit (cat. no. 7074) antibodies were obtained from Cell Signaling Technology, Inc. (Danvers, MA, USA). Chemicals for buffer 
preparations were purchased from Sigma-Aldrich (Merck $\mathrm{KGaA})$.

Cell line and cell culture. Human U87 glioma cells were obtained from the Cell Bank of the Chinese Academy of Sciences (Shanghai, China) and grown in DMEM containing $10 \%$ fetal bovine serum (Haoyang Biological Products Technology Co., Ltd., Tianjin, China), $100 \mathrm{U} / \mathrm{ml}$ penicillin, $100 \mu \mathrm{g} / \mathrm{ml}$ streptomycin, and incubated in a humidified atmosphere of $5 \% \mathrm{CO}_{2}$ at $37^{\circ} \mathrm{C}$.

Cell viability assay. Cell viability was assessed using an MTT bromide assay. U87 cells ( $200 \mu 1 ; 3 \times 10^{4}$ cells $\left./ \mathrm{ml}\right)$ were seeded into 96 -well tissue culture plates. Following overnight incubation, the cells were exposed to serial concentrations of SSd $(1,2,3,4,5,6,7$ and $8 \mu \mathrm{M})$ or control medium for $48 \mathrm{~h}$. Subsequently, $10 \mu \mathrm{l}$ MTT reagents was added to each well and incubated at $37^{\circ} \mathrm{C}$ for $4 \mathrm{~h}$, followed by the addition of $150 \mu \mathrm{l}$ dimethyl sulfoxide to each well. The plates were agitated for $10 \mathrm{~min}$. Then, the absorbance was read at a wavelength of $490 \mathrm{~nm}$ using an iMark ${ }^{\mathrm{TM}}$ microplate reader (Bio-Rad Laboratories, Inc., Hercules, CA, USA). Each experiment was performed with six replicate wells for each condition, and the data were obtained from three independent experiments. The half-maximal inhibitory concentration $\left(\mathrm{IC}_{50}\right)$ of SSd was calculated using the Logit method (15).

Hoechst 33258 staining and immunofluorescence. U87 cells in 6-well plates were treated with different concentrations of SSd for $48 \mathrm{~h}$, washed with phosphate-buffered saline (PBS) and fixed with $4 \%$ paraformaldehyde for $10 \mathrm{~min}$. Subsequently, the cells were washed with PBS, and stained with Hoechst 33258 for $5 \mathrm{~min}$ at room temperature. The nuclear morphology was observed using a laser scanning confocal microscope. For quantification, three different fields were randomly selected and counted under the microscope. The apoptotic rates were calculated as the percentage of apoptotic cells relative to the total number of cells.

Flow cytometric evaluation of apoptosis. Cells treated with different concentrations of SSd were seeded into 6-well plates $\left(1.8 \times 10^{5} \mathrm{cells} / \mathrm{well}\right)$ for $48 \mathrm{~h}$ and isolated with trypsin. The cells were supplemented with $100 \mu 1$ DMEM medium and analyzed according to the manufacturer's instructions using the Muse $^{\mathrm{TM}}$ Annexin V and Dead Cell Assay kit (Muse ${ }^{\mathrm{TM}}$ Cell Analyzer; Merck KGaA).

Western blotting. Following SSd treatment for 48 h, U87 cells were washed with ice-cold PBS three times and resuspended in $100 \mu \mathrm{l}$ radioimmune precipitation buffer $[100 \mathrm{mM} \mathrm{NaCl}$ and $100 \mathrm{mM}$ sodium fluoride, $20 \mathrm{mM}$ Tris-HCl (pH 7.4), $2.5 \mathrm{mM}$ EDTA, $1 \%$ SDS, $1 \%$ Triton X-100 and $1 \%$ sodium deoxycholate]. The lysate was centrifuged at $12,000 \mathrm{x}$ g for $20 \mathrm{~min}$, and $50 \mu \mathrm{g}$ cell lysate protein was used for western blotting. Proteins were separated by $10 \%$ SDS-PAGE and transferred to nitrocellulose membranes. Membranes were incubated overnight individually with primary antibodies at a dilution of 1:1,000 in TBS containing $0.1 \%$ Tween- 20 at $4^{\circ} \mathrm{C}$, and the membranes were incubated with peroxidase-conjugated anti-rabbit $\operatorname{IgG}$ $(1: 5,000)$ for $2 \mathrm{~h}$ at room temperature. All bands were detected

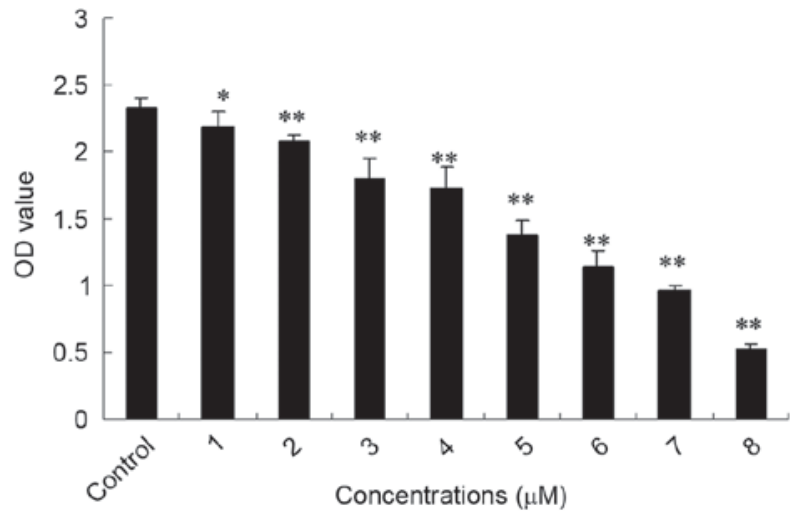

Figure 1. Effects of SSd exposure on the growth and proliferation of human U87 glioma cells in vitro. The U87 cells were treated with SSd at different concentrations for $48 \mathrm{~h}$. Cell viability rate was assayed by 3-(4,5-dimethylthiazol-2-yl)-2,5-diphenyltetrazolium. Data are presented as means \pm standard deviation. ${ }^{*} \mathrm{P}<0.05$ and ${ }^{* *} \mathrm{P}<0.01$ vs. control. SSd, saikosaponin $\mathrm{D}$; OD, optical density.

using the enhanced chemiluminescence (ECL) system (Tanon Science \& Technology Co., Ltd., Shanghai, China) according to the manufacturer's instructions (16).

Statistical analysis. The data are presented as the mean \pm standard deviations as indicated. Statistical analyses for comparison of mean values were performed by one-way analysis of variance followed by Dunnett's test. $\mathrm{P}<0.05$ was considered to indicate a statistically significant difference. All data were derived from three independent experiments.

\section{Results}

SSd treatment inhibited the proliferation of U87 cells. The mitochondria of living cells break down MTT to produce formazan, and the quantity of formazan corresponds with the living cell number. To determine whether SSd inhibits U87 glioma cell growth, the cell viability rate was assessed using the MTT method. In the present study, obvious inhibitory effects on the proliferation of U87 cells were observed with SSd exposure. As shown in Fig. 1, following treatment with 1-8 $\mu \mathrm{M}$ SSd for $48 \mathrm{~h}$, the proliferation rate of U87 cells was significantly reduced in a dose-dependent manner when compared with the control group. Additionally, the half maximal inhibitory concentration $\left(\mathrm{IC}_{50}\right)$ value of $\mathrm{SSd}$ was $4.79 \mu \mathrm{M}$. The result indicates that SSd inhibited the viability of U87 glioma cells.

Effect of SSd treatment on changes of cellular morphology. When detecting nuclear morphological changes, U87 cells were stained with Hoechst 33258 and observed under a laser scanning confocal microscope. Intact nuclei in the living cells were stained blue, and the morphology was round or oval, while the condensed or fragmented nuclei in apoptotic cells were stained bright blue. As illustrated in Fig. 2, the cells treated with $2.5 \mu \mathrm{M}$ SSd began to exhibit nuclear morphological changes when compared with the control cells, and the nuclei of cells treated with $7.5 \mu \mathrm{M} \mathrm{SSd}$ were markedly brighter, indicating a high prevalence of nuclear chromatin and fragmentation. 
A

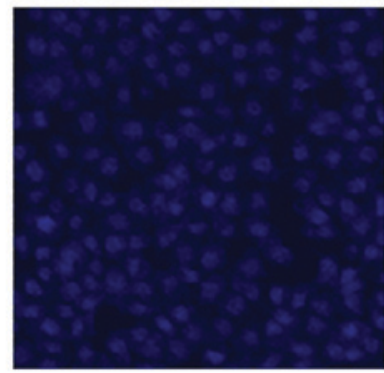

Control

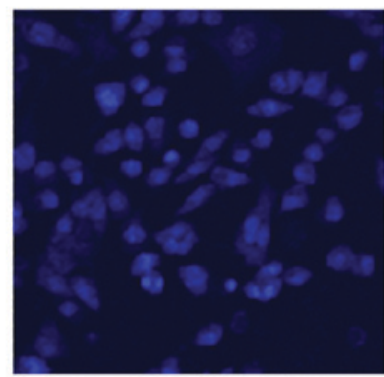

$5 \mu \mathrm{M}$

B

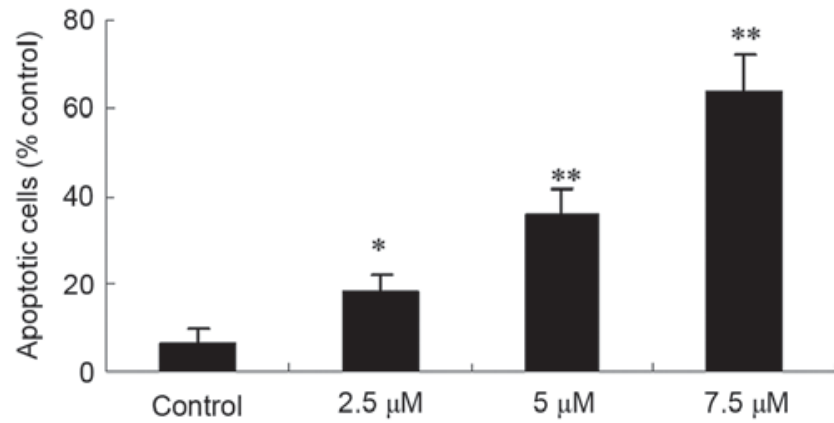

Figure 2. Hoechst 33258 staining of U87 cells observed under a laser scanning confocal microscope following treatment with saikosaponin D at different concentrations for $48 \mathrm{~h}$ (magnification, x200). (A) Nuclear morphological changes in each group were determined by Hoechst 33258 staining. Highly fragmented or condensed nuclei represent apoptotic cells and intact nuclei represent viable cells. (B) Bar graphs represent the percentage of nuclear fragments and condensation in the U87 cells. Data are presented as means \pm standard deviation $(n=3)$. ${ }^{*} \mathrm{P}<0.05$ and ${ }^{* *} \mathrm{P}<0.01$ vs. control.

SSd treatment induced apoptosis in U87 cells. To further evaluate whether the inhibition of cell proliferation in U87 cells was associated with the induction of apoptosis, Annexin V staining was used to assess the rate of apoptotic cells following SSd treatment. As shown in Fig. 3, treatment with SSd significantly increased the proportion of Annexin $\mathrm{V}$-positive cells in a dose-dependent manner. The results indicate that SSd treatment inhibited the viability of U87 cells by inducing apoptosis.

Effect of SSd treatment on phosphatidylinositol 3-kinases $(P I 3 K) / A k t, E R K$ and JNK signaling pathways in U87 cells. To further elucidate the possible mechanisms underlying the effects of SSd treatment on U87 cells, the p-Akt/Akt, p-ERK/ERK, p-JNK/JNK protein expression levels with western blot analysis. The data demonstrated that SSd exposure induced a significant decrease of p-Akt and p-ERK
A

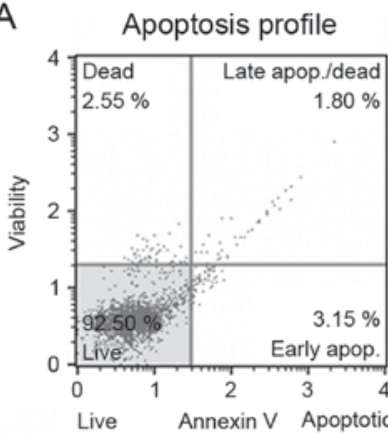

Contro
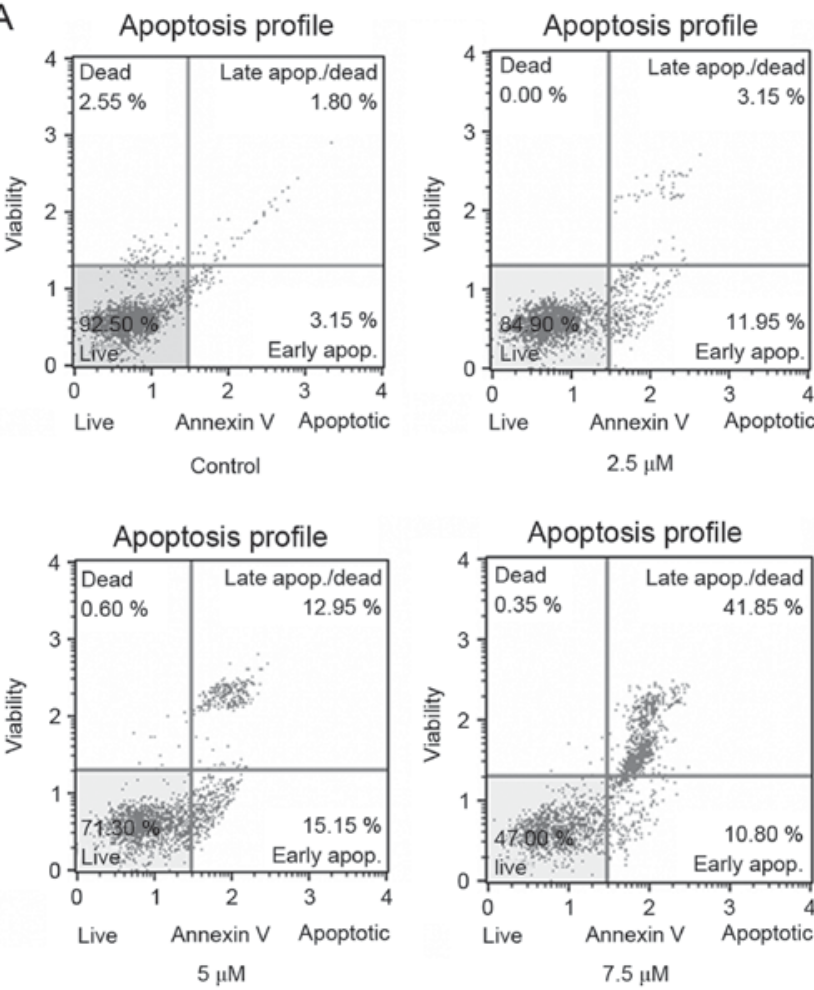

B

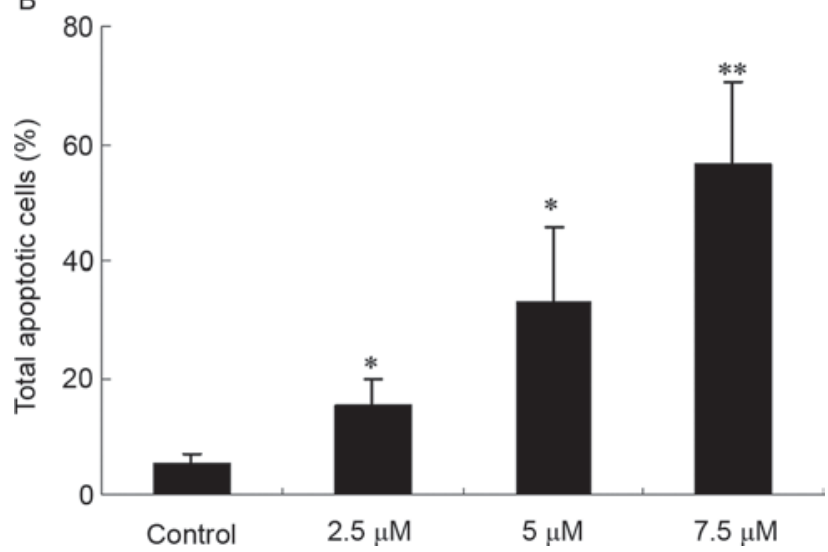

Figure 3. Effects of saikosaponin D treatment on the apoptotic rate of U87 cells as evaluated by Muse ${ }^{\mathrm{TM}}$ Annexin V and Dead Cell assay. (A) Muse ${ }^{\mathrm{TM}}$ Cell Analyzer analysis of Annexin V-stained cells. (B) Quantitative analysis of data from the total apoptotic cell ratio. Data are presented as means \pm standard deviation $(\mathrm{n}=3)$. ${ }^{*} \mathrm{P}<0.05$ and ${ }^{* *} \mathrm{P}<0.01$ vs. control.

relative protein expression levels, and increased $\mathrm{p}-\mathrm{JNK}$ protein expression levels (Fig. 4; $\mathrm{P}<0.05$ ). These results indicated that treatment with $\mathrm{SSd}$ potentially downregulated the PI3K/Akt and ERK signaling pathways, increased JNK activation and further enhanced apoptosis in U87 cells.

Activation of caspase-3 induced by SSd exposure. Caspases are critical mediators during the process of apoptosis. In all of apoptosis-associated caspases, caspase-3 is a key effector or executioner for programmed cell death (17). Thus, to determine whether caspase-3 was involved in SSd-induced cytotoxicity, the expression levels of cleaved caspase-3, an indicator of its activity, were examined. In the present study, SSd treatment significantly increased the expression level of cleaved caspase-3 (Fig. 5), indicating that SSd-induced 
A
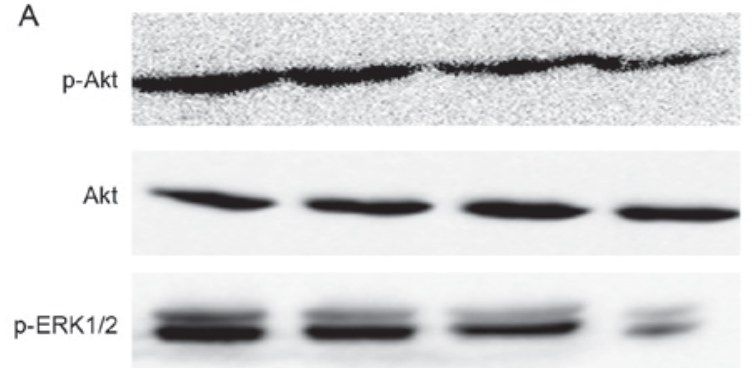

ERK1/2

p-JNK1/2

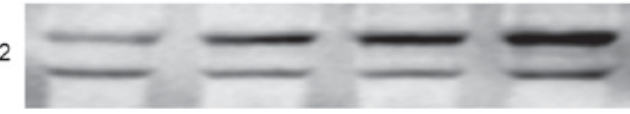

JNK1/2

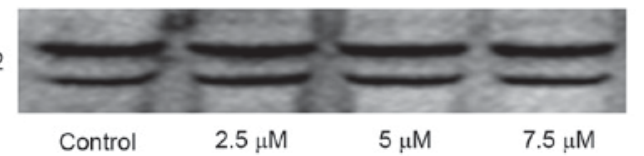

B

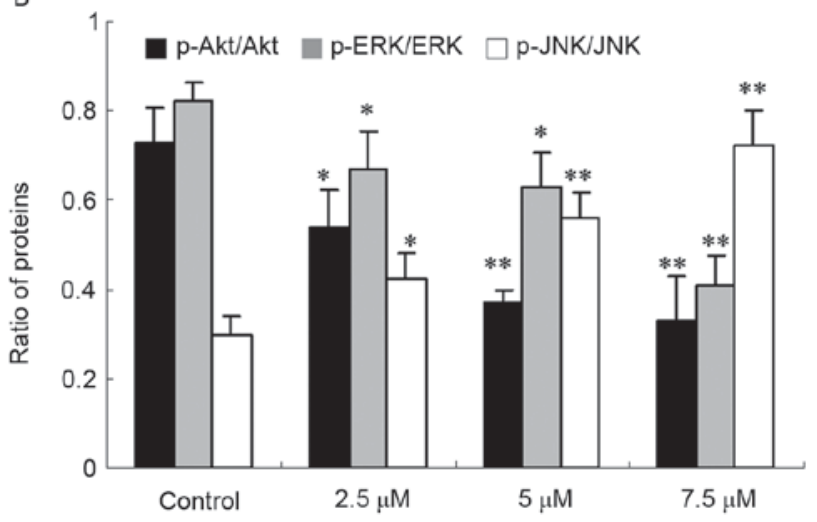

Figure 4. Effects of SSd treatment on phosphorylated and total Akt, ERK and JNK protein expression levels. (A) Western blot analysis of phosphorylated and total Akt, ERK and JNK levels with SSd administration at different concentrations for $48 \mathrm{~h}$. Bands were visualized using the ECL detection method. (B) Relative expression levels of p-Akt/Akt, p-ERK/ERK and $\mathrm{p}-\mathrm{JNK} / \mathrm{JNK}$ ratios. Data are presented as means \pm standard deviation $(\mathrm{n}=3)$. ${ }^{*} \mathrm{P}<0.05$ and ${ }^{* *} \mathrm{P}<0.01$ vs. control. SSd, saikosaponin D; ERK, extracellular signal-regulated kinases; JNK, c-Jun N-terminal kinases; p, phosphorylated.

apoptosis in U87 cells may be associated with the promoted activation of caspase-3.

\section{Discussion}

As a traditional Chinese medicine, B. falcatum $L$ is commonly administered for the treatment of hepatopathy, inflammation and viral infection in Asia. Triterpene saponins are the major pharmaceutical ingredients in B. falcatum $L$, which are divided into saikosaponin-a, $-b$, $-c$ and $-d$ according to the different structures. SSd is one of the most active ingredients of triterpene saponins. During the past decades, studies have focused on SSd, as it demonstrates numerous beneficial properties, including anti-inflammatory, antioxidant and antitumor effects $(12,18,19)$. In the present study, it was demonstrated that SSd treatment inhibited the proliferation of human malignant glioma U87 cells, indicating that SSd may exert potential beneficial effects in the treatment of malignant gliomas.
A
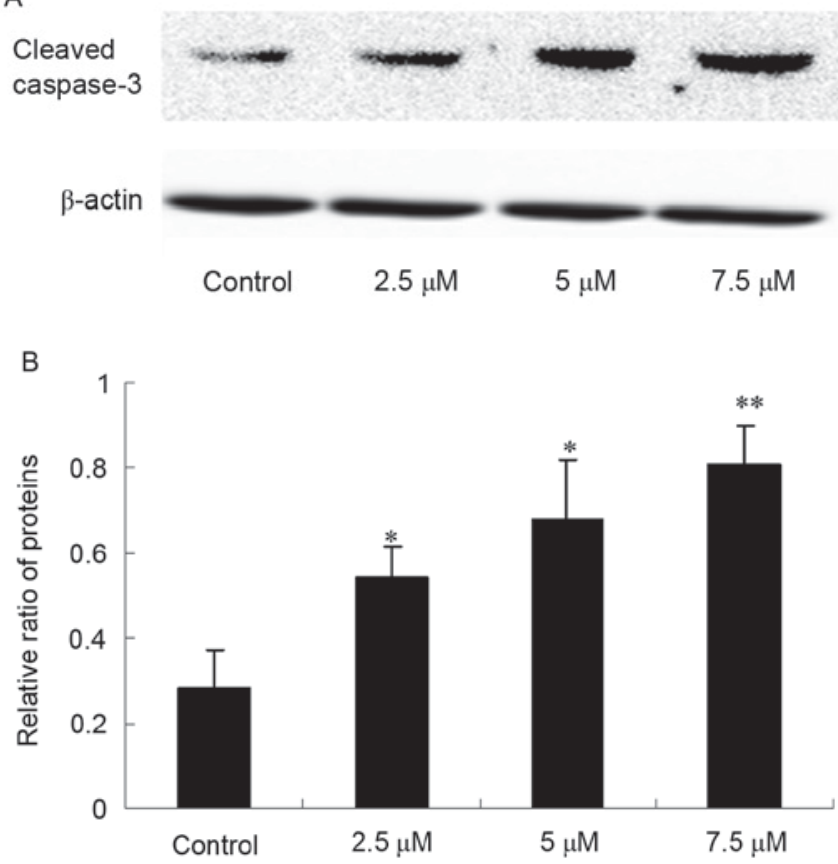

Figure 5. Saikosaponin D treatment upregulated the activity of caspase-3. (A) Cleaved caspase-3 expression levels were assayed by western blotting. (B) Relative densitometric analysis of the gels presented in (A). Data shown are representative of three different experiments. Values are presented as means \pm standard deviation of three separate experiments. ${ }^{*} \mathrm{P}<0.05$ and ${ }^{* * *} \mathrm{P}<0.01$ vs. control.

Apoptosis is recognized as the most important form of cell death in multicellular organisms, and occurs in physiological and pathological conditions. Certain types of cytotoxic stresses, such as hypoxia, UV, infared irradiation and chemotherapeutic drugs, initiate apoptosis to remove target cells $(20,21)$. Biochemical alterations of apoptosis include phosphatidylserine externalization, chromosomal DNA cleavage, and activation of a family of proteases (22). Apoptosis is important for the inhibition of cancer development $(23,24)$. At present, drug-induced apoptosis is considered to be the primary strategy for curing tumors (25). Previous studies have confirmed that SSd exposure inhibits proliferation and promotes apoptosis in certain tumor cells (26-28). However, whether SSd induces apoptosis in glioma cells has yet to be elucidated. In the present study, the addition of SSd led to increased apoptosis in human U87 glioma cells, as measured by Hoechst 33258 staining and Annexin V staining assays, implying that SSd treatment exerts significant cytotoxic effects by increasing apoptosis in glioma cells.

In the current study, it was also found that SSd inhibited the PI3K/Akt signaling pathway in a dose-dependent manner, indicating that depression of the PI3K/AKT signaling pathway may be associated with SSd-mediated apoptosis and proliferation inhibition. Akt, also known as protein kinase $\mathrm{B}$, is a primary downstream effector of the PI3K signal transduction pathway, with crucial functions in regulating cell proliferation and survival $(29,30)$. Furthermore, it has been reported that the Akt signaling pathway is particularly important in preventing apoptosis $(31,32)$ and inhibiting the activation of Akt may induce apoptosis (33). ERK and JNK are members of the mitogen-activated protein kinase 
(MAPK) family, and have been shown to be key in cell proliferation, differentiation, development and programmed cell death (34). Numerous studies have indicated that abnormalities in MAPK signaling pathways were involved in the pathological processes of various types of cancer (35-37). In the current study, the aim was to investigate the effects of SSd exposure on the protein expression levels of ERK and JNK. The results demonstrated that SSd treatment significantly inhibited the activation of ERK and stimulated the phosphorylation of JNK.

There are three apoptotic pathways found in mammals: The extrinsic pathway (death receptor-mediated pathway), the intrinsic pathway (mitochondrial-mediated pathway) and the granzyme B pathway. The key regulatory factors in these three pathways are the caspases (cysteinyl aspartate-specific proteinases), which are activated following cell damage and are responsible for regulating cell apoptosis (38). Accordingly, in recent years, much attention has been paid to developing anticancer therapeutic strategies that modulate the activation of caspases to inhibit tumor progression. Caspase- 3 is a major mediator during the execution period of cell apoptosis. When activated by upstream signaling molecules, such as caspase-8, caspase-9 or caspase-10, caspase- 3 would be either partially or totally responsible for the proteolysis of many downstream key proteins associated with apoptosis (39). In order to elucidate the molecular mechanisms responsible for SSd-induced apoptosis in U87 cells, the activity of caspase-3 was analyzed in the present study. The findings indicated that the addition of SSd to U87 cells promoted caspase-3 activity, demonstrating the role of capase- 3 activation in $\mathrm{SSd}$-induced apoptosis.

In conclusion, the present study demonstrated that SSd treatment in human glioma cells inhibits cell proliferation, downregulates phosphorylation of Akt and ERK, upregulates JNK and caspase-3 activities and eventually causes cell apoptosis. Collectively, these observations provide further understanding of the pharmacological activity of SSd. However, future work is required to elucidate the underlying mechanisms of SSd-induced tumor cell apoptosis.

\section{Acknowledgements}

The present study was supported by the Department of Education of Jilin Province (grant no. 2015407) and Project of Science \& Technology Development of Jilin Province (grant no. 201205079).

\section{References}

1. Crocetti E, Trama A, Stiller C, Caldarella A, Soffietti R, Jaal J, Weber DC, Ricardi U, Slowinski J and Brandes A; RARECARE working group: Epidemiology of glial and non-glial brain tumours in Europe. Eur J Cancer 48: 1532-1542, 2012.

2. Ricard D, Idbaih A, Ducray F, Lahutte M, Hoang-Xuan K and Delattre JY: Primary brain tumours in adults. Lancet 379: 1984-1996, 2012.

3. de-Almeida-Sassi F, Lunardi-Brunetto A, Schwartsmann G, Roesler R and Abujamra AL: Glioma revisited: From neurogenesis and cancer stem cells to the epigenetic regulation of theniche. J Oncol 2012: 537861, 2012.

4. Lima FR, Kahn SA, Soletti RC, Biasoli D, Alves T, da Fonseca AC, Garcia C, Romão L, Brito J, Holanda-Afonso R, et al: Glioblastoma: Therapeutic challenges, what lies ahead. Biochim Biophys Acta 1826: 338-349, 2012.
5. Shahar T, Nossek E, Steinberg DM, Rozovski U, Blumenthal DT, Bokstein F, Sitt R, Freedman S, Corn BW, Kanner AA and Ram Z: The impact of enrollment in clinical trials on survival of patients with glioblastoma. J Clin Neurosci 19: 1530-1534, 2012.

6. Shao J, Zheng D, Jiang Z, Xu H, Hu Y, Li X and Lu X: Curcumin delivery by methoxy polyethylene glycol-poly(caprolactone) nanoparticles inhibits the growth of C6 glioma cells. Acta Biochim Biophys Sin (Shanghai) 43: 267-274, 2011.

7. Wang YB, Hu Y, LiZ, Wang P, Xue YX, Yao YL, Yu B and Liu YH: Artemether combined with shRNA interference of vascular cell adhesion molecule-1 significantly inhibited the malignant biological behavior of human glioma cells. PLoS One 8: e60834, 2013.

8. Zhang FY, Hu Y, Que ZY, Wang P, Liu YH, Wang ZH and Xue YX: Shikonin inhibits the migration and invasion of human glioblastoma cells by targeting phosphorylated $\beta$-catenin and phosphorylated PI3K/Akt: A potential mechanism for the anti-glioma efficacy of a traditional chinese herbal medicine. Int J Mol Sci 16: 23823-23848, 2015.

9. Tundis R, Bonesi M, Deguin B, Loizzo MR, Menichini F, Conforti F, Tillequin F and Menichini F: Cytotoxic activity and inhibitory effect on nitric oxide production of triterpene saponins from the roots of Physospermum verticillatum (Waldst \& Kit) (Apiaceae). Bioorg Med Chem 17: 4542-4547, 2009.

10. Wang Q, Zheng XL, Yang L, Shi F, Gao LB, Zhong YJ, Sun H, $\mathrm{He} F$, Lin $\mathrm{Y}$ and Wang $\mathrm{X}$ : Reactive oxygen species-mediated apoptosis contributes to chemosensitization effect of saikosaponins on cisplatin-induced cytotoxicity in cancer cells. J Exp Clin Cancer Res 29: 159, 2010.

11. Wang BF, Lin S, Bai MH, Song LQ, Min WL, Wang M, Yang P, $\mathrm{Ma} \mathrm{HB}$ and Wang XJ: Effects of SSd combined with radiation on inhibiting SMMC-7721 hepatoma cell growth. Med Sci Monit 20: 1340-1344, 2014.

12. Wong VK, Li T, Law BY, Ma ED, Yip NC, Michelangeli F, Law CK, Zhang MM, Lam KY, Chan PL and Liu L: Saikosaponin-d, a novel SERCA inhibitor, induces autophagic cell death in apoptosis-defective cells. Cell Death Dis 4: e720, 2013.

13. Wong VK, Zhang MM, Zhou H, Lam KY, Chan PL, Law CK, Yue PY and Liu L: Saikosaponin-d enhances the anticancer potency of TNF- $\alpha$ via overcoming its undesirable response of activating NF-kappa B signalling in cancer cells. Evid Based Complement Alternat Med 2013: 745295, 2013.

14. Hsu YL, Kuo PL, Chiang LC and Lin CC: Involvement of p53, nuclear factor kappaB and Fas/Fas ligand in induction of apoptosis and cell cycle arrest by saikosaponin $d$ in human hepatoma cell lines. Cancer Lett 213: 213-221, 2004.

15. Acharya AS, Acharya NK and Dash AP: Software for estimating LD50 and LD90 by logit analysis. Comput Methods Programs Biomed 34: 255-256, 1991.

16. Nesbitt SA and Horton MA: A nonradioactive biochemical characterization of membrane proteins using enhanced chemiluminescence. Anal Biochem 206: 267-272, 1992.

17. Porter AG and Jänicke RU: Emerging roles of caspase-3 in apoptosis. Cell Death Differ 6: 99-104, 1999.

18. Lin X, Wu S, Wang Q, Shi Y, Liu G, Zhi J and Wang F: Saikosaponin-D reduces $\mathrm{H} 2 \mathrm{O} 2$-induced $\mathrm{PC} 12$ cell apoptosis by removing ROS and blocking MAPK-dependent oxidative damage. Cell Mol Neurobiol 36: 1365-1375, 2016.

19. Wang P, Ren J, Tang J, Zhang D, Li B and Li Y: Estrogen-like activities of saikosaponin-d in vitro: A pilot study. Eur J Pharmacol 626: 159-165, 2010.

20. Elmore S: Apoptosis: A review of programmed cell death. Toxicol Pathol 35: 495-516, 2007.

21. Kerr JF, Wyllie AH and Currie AR: Apoptosis: A basic biological phenomenon with wide-ranging implications in tissue kinetics. Br J Cancer 26: 239-257, 1972.

22. Ouyang L, Shi Z, Zhao S, Wang FT, Zhou TT, Liu B and Bao JK: Programmed cell death pathways in cancer: A review of apoptosis, autophagy and programmed necrosis. Cell Prolif 45: 487-498,2012.

23. Kin KL and Cidlowski JA: Cell cycle regulation and apoptosis. Annu Rev Physiol 60: 601-617, 1998.

24. Townson JL, Naumov GN and Chambers AF: The role of apoptosis in tumor progression and metastasis. Curr Mol Med 3: 631-642, 2003.

25. Saddoughi SA, Song P and Ogretmen B: Roles of bioactive sphingolipids in cancer biology and therapeutics. Subcell Biochem 49: 413-440, 2008

26. He S, Lu G, Hou H, Zhao Z, Zhu Z, Lu X, Chen J and Wang Z: Saikosaponin-d suppresses the expression of cyclooxygenase-2 through the phospho-signal transducer and activator of transcription 3/hypoxia-inducible factor-1 $\alpha$ pathway in hepatocellular carcinoma cells. Mol Med Rep 10: 2556-2562, 2014. 
27. Jang MJ, Kim YS, Bae EY, Oh TS, Choi HJ, Lee JH, Oh HM and Lee SW: Saikosaponin D isolated from Bupleurum falcatum inhibits selectin-mediated cell adhesion. Molecules 19: 20340-20349, 2014.

28. Liu RY and Li JP: Saikosaponin-d inhibits proliferation of human undifferentiated thyroid carcinoma cells through induction of apoptosis and cell cycle arrest. Eur Rev Med Pharmacol Sci 18: 2435-2443, 2014.

29. Davis WJ, Lehmann PZ and Li W: Nuclear PI3K signaling in cell growth and tumorigenesis. Front Cell Dev Biol 3: 24, 2015.

30. Wymann MP, Zvelebil M and Laffargue M: Phosphoinositide 3 kinase signalling-which way to target? Trends Pharmacol Sci 24: 366-376, 2003.

31. OuYang F, Wang G, Guo W, Zhang Y, Xiang W and Zhao M: AKT signalling and mitochondrial pathways are involved in mushroom polysaccharide-induced apoptosis and G1 or S phase arrest in human hepatoma cells. Food Chem 138: 2130-2139, 2013.

32. Warfel NA and Kraft AS: PIM kinase (and Akt) biology and signaling in tumors. Pharmacol Ther 151: 41-49, 2015.
33. Nakagawa A, Sullivan KD and Xue D: Caspase-activated phosphoinositide binding by CNT-1 promotes apoptosis by inhibiting the AKT pathway. Nat Struct Mol Biol 21: 1082-1090, 2014.

34. Widmann C, Gibson S, Jarpe MB and Johnson GL: Mitogenactivated protein kinase: Conservation of a three-kinase module from yeast to human. Physiol Rev 79: 143-180, 1999.

35. Koul HK, Pal M and Koul S: Role of p38 MAP kinase signal transduction in solid tumors. Genes Cancer 4: 342-359, 2013.

36. Gkouveris I, Nikitakis N, Karanikou M, Rassidakis G and Skavounou A: JNK1/2 expression and modulation of STAT3 signaling in oral cancer. Oncol Lett 12: 699-706, 2016.

37. Wang Z, Wang W, Xu S, Wang S, Tu Y, Xiong Y, Mei J and Wang C: The role of MAPK signaling pathway in the Her-2-positive meningiomas. Oncol Rep 36: 685-695, 2016.

38. Jin $\mathrm{Z}$ and El-Deiry WS: Overview of cell death signaling pathways. Cancer Biol Ther 4: 139-163, 2005.

39. Mazumder S, Plesca D and Almasan A: Caspase-3 activation is a critical determinant of genotoxic stress-induced apoptosis. Methods Mol Biol 414: 13-21, 2008. 